\title{
Towards Situation Awareness in Integrated Air Defence Using Clustering and Case Based Reasoning
}

\author{
Manish Gupta and Sumant Mukherjee \\ Institute for Systems Studies and Analyses \\ Defence Research \& Development Organisation \\ Metcalfe House, Delhi. India 110054 \\ manish.iitdelhi@gmail.com
}

\begin{abstract}
For integrated air defence in network centric environment, it is required to process information (collected from multiple sensors) for enhancing Situation Awareness (SA) at the command and control nodes. SA is the process of building comprehensive pictures of the battlespace to the decision maker who can further utilized it for threat evaluation. A novel approach for enhancing situation awareness in integrated air defence perspective via Clustering and Case Based Reasoning (CBR) has been proposed in this paper. Clustering is applied on track data generated from Level I of multi sensor data fusion to aggregate entities in the target area. CBR further provide information about air package type, size and purpose of the aggregated entities using cluster attribute records. The effectiveness of the proposed approach has been illustrated on simulation data generated to depict typical integrated defence scenario.
\end{abstract}

Keywords: Network Centric Warfare, Integrated Air Defence, Situation Awareness, Clustering, Case Based Reasoning.

\section{Introduction}

Network Centric Warfare (NCW) 2 is the term used in military circles to define information-based war fighting. Network centric battle space can be visualized as network of sensors, platforms and operators (military forces) and Command \& Control (C2) entities where free flow of information takes place unlike stovepipe flow of conventional C2 hierarchy.For NCW, data from multiple sources is collected and fused to create the 'Common Operational Picture' (COP) 7/8] using various techniques of Multi-Sensor Data Fusion (MSDF) 10.MSDF is an evolving technology, concerning the problem of how to fuse data from multiple sensors intelligently in order to make a more accurate estimation of the environment. Applications of data fusion cross a wide spectrum, including environment monitoring, automatic target detection and tracking, battlefield surveillance, remote sensing, situation awareness, etc. 
Situation Awareness (SA) 34] was envisioned as the main part of Level 2 processing in the JDL model of MSDF 10]. Assuming that object identification has been done at Level I, Level II tries to understand situation by grouping identified objects and interpreting patterns emerging out of this grouping. Essentially four processes take place at this stage object aggregation, event aggregation, context interpretation and multi-perspective assessment. Pattern recognition techniques and automated reasoning techniques are used to implement these processes.

The paper provides a novel approach for enhancing situation awareness in integrated air defence perspective via Clustering [5] and Case Based Reasoning (CBR) 169. Clustering is applied outputs generated from level I of MSDF to aggregate entities in the target area. CBR further provide information about unit type, unit size and unit purpose of the aggregated entities using cluster attribute records. The effectiveness of the proposed approach has been illustrated on simulation data generated to depict typical integrated defence scenario.

Section 2 provides an overview of Situation Awareness (SA) and its components. Proposed approach along with application scope of clustering and case based reasoning in SA is described in section 3. Section 4 shows the simulation experiments to illustrate the effectiveness of the proposed approach. Concluding remarks with future scope of the study are given in the last section.

\section{Situation Awareness: An Overview}

Situation awareness has been formally defined by Endsley 3 as "the perception of elements in the environment within a volume of time and space, the comprehension of their meaning, and the projection of their status in the near future". Situation Awareness (SA) involves being aware of what is happening around you to understand how information, events, and your own actions will impact your goals and objectives, both now and in the near future and deriving relations among entities, e.g., the aggregation of object states (i.e., classification and location).

\subsection{Endsley's Model}

Endsley 3] specifies three levels of Situation Awareness namely, Perception, comprehension and projection. The first step in achieving SA is to perceive the status, attributes, and dynamics of relevant elements in the environment. Thus, Level 1 SA leads to an awareness of multiple situational elements (objects, events, people, systems, environmental factors) and their current states (locations, conditions, modes, actions). Level $2 \mathrm{SA}$ integrate disjointed Level 1 information through the processes of pattern recognition to understand how it will impact upon the individual's goals and objectives. This includes developing a comprehensive picture of the battlefield. Level $3 \mathrm{SA}$ is achieved through knowledge of the status and dynamics of the elements and comprehension of the situation (Levels 1 and $2 \mathrm{SA}$ ), and then extrapolating this information forward in time to determine how it will affect future states of the operational environment. 


\section{Proposed Approach}

The paper proposes a unique approach based on clustering and case based reasoning to achieve situation awareness for integrated air defence environment. The flow diagram of the proposed approach for target detection and situation awareness is shown in Figure 1. It is two stage processes in which targets are aggregated geographically using hierarchical clustering [5] and aggregated objects are further evaluated to build common operational picture using CBR. Feature vector is extracted on the output data of Level-I of MSDF. Clusters are formed based on the position of the targets which further generates cluster attribute records. The cluster attribute records are further matched using CBR technique with the existing CBR library to determine air package size, type and purpose. The details of the proposed approach are given in the following subsections.

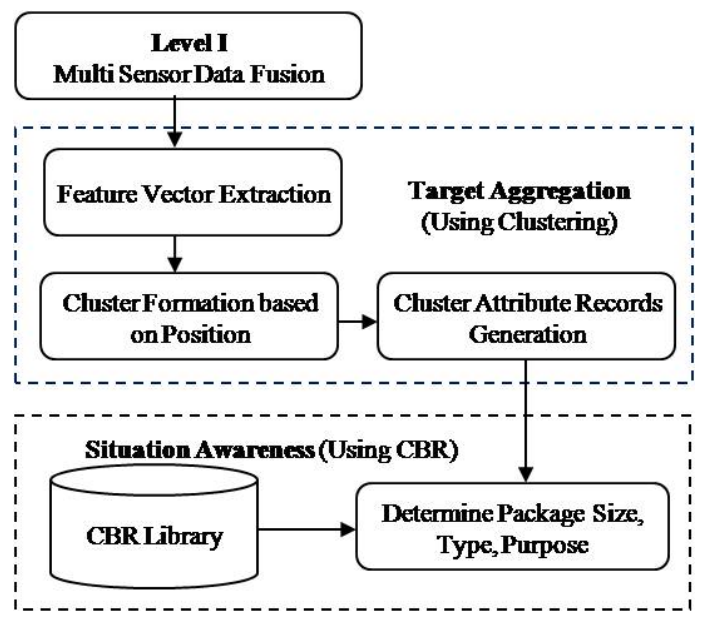

Fig. 1. Proposed Approach for Target Aggregation and Situation Awareness

\subsection{Clustering Algorithm for Targets Aggregation}

The feature vector obtained from Level-I of MSDF for each air target contains the information such as (x,y,z)-position components and the class beliefs are between 0.0 and 1.0. The feature vectors for a battlespace are to be updated from tracks at certain times to yield a current set of target feature vectors for SA processing. The clustering of these vectors is done only with respect to position features $(\mathrm{x}, \mathrm{y}, \mathrm{z})$ in a given area of 20x20x1 kilometers.

Once clusters are for formed as target aggregation using hierarchical clustering [5], cluster attribute records are generated by considering number of individual target type and their average class beliefs as weight for every cluster. Cluster attribute records serve as the problem part for further analysis of Casebased reasoning $(\mathrm{CBR})$. The problem is to be compared to the problem parts of 
cases in the CBR library to extract a similar case whose solution is in the form of air package type, size, and purpose of the cluster.

\subsection{Case Based Reasoning for Building Common Operational Picture}

Case-based reasoning (CBR) by Roger Schank 9 in early 1980s is the process of solving new problems based on the solutions of similar past problems. Generally, CBR is regarded as a vital approach when it is difficult to formulate rules describing the situations e.g. car repairing by Mechanic, Lawyer etc. It is a four step process namely retrieve, reuse, revise and retain.

Dynamic memory model 96 has been used for case storage in CBR Library. Each case in the SA case-base is made up of problem and solution parts. The new cluster attribute record is compared with the problem parts of cases of CBR library and extract solution of a similar case using case retrieval methods such as nearest neighbor, induction and template retrieval. We have applied the nearest neighbor method, which compares the similarity between the cases of CBR library and the input problem. The case similarity calculation uses the information of quantity proximity, the degree of belief, and the weight of importance of each target class. One simulation study was carried out to illustrate the effectiveness of the proposed approach in integrated air defence scenario.

\section{Simulation Results}

The proposed approach has been implemented using simulation study. Table 1 shows the feature vector of ten targets obtained from Level I of MSDF. Feature vector shows the positional vector of the targets along with class and its class belief calculated at identity estimation algorithm of Level I. Time and date can also used for dynamic case of creating common operational picture (COP). The last column of the Table 1 shows the cluster index of the targets using hierarchical clustering. The clustering results are described in further subsection.

Table 1. Feature Vector of Tracked Data and Cluster Index

\begin{tabular}{|c|c|c|c|c|c|c|c|c|}
\hline Tgt No. & Time & Date & $\mathrm{X}$ & $\mathrm{Y}$ & $\mathrm{Z}$ & Class & Class Belief & Cluster Index \\
\hline 1 & 14.01 & $14 / 4 / 2009$ & 2092 & 3451 & 15009 & 2 & 0.7 & 1 \\
2 & 14.01 & $14 / 4 / 2009$ & 2083 & 3480 & 14500 & 1 & 0.8 & 1 \\
3 & 14.01 & $14 / 4 / 2009$ & 2507 & 3076 & 15890 & 2 & 0.7 & 1 \\
4 & 14.01 & $14 / 4 / 2009$ & 2465 & 2946 & 16879 & 1 & 0.6 & 2 \\
5 & 14.01 & $14 / 4 / 2009$ & 2345 & 3215 & 12036 & 2 & 0.7 & 3 \\
6 & 14.01 & $14 / 4 / 2009$ & 2106 & 3514 & 14569 & 3 & 0.8 & 1 \\
7 & 14.01 & $14 / 4 / 2009$ & 2365 & 3489 & 13568 & 1 & 0.7 & 4 \\
8 & 14.01 & $14 / 4 / 2009$ & 2678 & 3012 & 15442 & 2 & 0.6 & 1 \\
9 & 14.01 & $14 / 4 / 2009$ & 1817 & 3489 & 17899 & 3 & 0.7 & 5 \\
10 & 14.01 & $14 / 4 / 2009$ & 1716 & 3128 & 16454 & 2 & 0.7 & 2 \\
\hline
\end{tabular}




\subsection{Clustering Results}

Hierarchical clustering has been applied to obtain clusters of the given targets. Threshold is also obtained the $70 \%$ of the maximum distance between the targets. It is observed that five clusters are obtained for the simulation data. Cluster attribute records are further generated by considering number of individual target type and their average class beliefs as weight for every cluster. Table 2 shows the cluster attribute records of the all five clusters obtained using clustering. CBR are further applied on the cluster attribute records for obtaining the best match to determine package type, size and purpose from the CBR library. The subsequent section describes the CBR procedure in detail.

Table 2. Cluster Attribute Records

\begin{tabular}{|c|c|c|c|c|c|c|c|c|}
\hline $\begin{array}{c}\text { Type 1 } \\
\text { Tgts No. }\end{array}$ & $\begin{array}{c}\text { Type 1 } \\
\text { Tgts \% }\end{array}$ & $\begin{array}{c}\text { Type 1 } \\
\text { Weight }\end{array}$ & $\begin{array}{c}\text { Type 2 } \\
\text { Tgts No. }\end{array}$ & $\begin{array}{c}\text { Type 2 } \\
\text { Tgts \% }\end{array}$ & $\begin{array}{c}\text { Type 2 } \\
\text { Weight }\end{array}$ & $\begin{array}{c}\text { Type 3 } \\
\text { Tgts No. }\end{array}$ & $\begin{array}{c}\text { Type 3 } \\
\text { Tgts \% }\end{array}$ & $\begin{array}{c}\text { Type 3 } \\
\text { Weight }\end{array}$ \\
\hline 1 & 20 & 0.8 & 3 & 60 & 0.67 & 1 & 20 & 0.8 \\
1 & 50 & 0.6 & 1 & 50 & 0.7 & 0 & 0 & 0 \\
0 & 0 & 0 & 1 & 100 & 0.7 & 0 & 0 & 0 \\
1 & 100 & 0.7 & 0 & 0 & 0 & 0 & 0 & 0 \\
0 & 0 & 0 & 0 & 0 & 0 & 1 & 100 & 0.7 \\
\hline
\end{tabular}

\subsection{CBR Results}

Case Base Reasoning (CBR) Library is required for applying CBR on cluster attribute records. The library records contain two part i.e. problem part and solution part. The problem part contains the same fields given in the cluster attribute records. The solution part consists of package type (e.g. bomber, fighter etc), package size (e.g. single, Multiple \& Squadron etc) and purpose (Defensive, Offensive, Surveillance etc.). Table 3 shows the solution part for all five clusters obtained by matching the most similar case of the CBR Library using standard similarity matrix. The CBR results can further be used for creating common operational picture (COP) and threat evaluation at MSDF Level III. It is to be noted that solution part can be different for different application. It can be Lethality and Attack Profile of the target but similar CBR Library is prerequisite for such study.

Table 3. CBR Results

\begin{tabular}{|c|c|c|c|}
\hline Cluster No. & Package Type & Package Size & Package Purpose \\
\hline 1 & Fighter A/c & Multiple(3) & Offensive \\
2 & Multi Role A/c & Unknown & Surveillance \\
3 & Fighter A/c & Multiple(3) & Offensive \\
4 & Multi Role A/c & Multiple(5) & Defensive \\
5 & Multi Role A/c & Unknown & Surveillance \\
\hline
\end{tabular}




\section{Concluding Remarks}

The paper proposes a novel approach of enhancing situation awareness using clustering and case based reasoning (CBR). The use of clustering depicts its utility for target aggregation for Integrated Air Defence in Network Centric Warfare (NCW). The role of CBR highlights to create Common Operational Picture (COP), which is further assessed by commanders for threat evaluation and for taking preventive measures in the battlefield scenario. From the simulation results is can be concluded that proposed approach can be effectively applied for various application in situation awareness. The proposed approach will be applied in the dynamic scenario as a future work.

Acknowledgments. We are highly indebted to Shri H.V. Srinivasa Rao, Director, ISSA for his active support and guidance. We are also thankful to Smt. Sanchita Malik, Scientist E, ISSA and our colleagues for sharing their valuable information in this field.

\section{References}

1. Aamodt, A.: Explanation-driven case-based reasoning, Topics in Case-based reasoning, pp. 274-288. Springer, Heidelberg (1994)

2. Ahlberg, S., et al.: An information fusion demonstrator for tactical intelligence processing in network-based defense. Information Fusion 8, 84-107 (2007)

3. Endsley, M.R.: Toward a theory of situational awareness in dynamic systems. Human Factors 37, 32-64 (1995)

4. Feng, H., et al.: Modelling situation awareness for Context-aware Decision Support. Expert Systems with Applications 36, 455-463 (2009)

5. Johnson, S.C.: Hierarchical clustering schemes. Psychometrika 32(3), 241-254 (1967)

6. Kolodner, J.L.: Maintaining Organization in a Dynamic Long-Term Memory. Cognitive Science 7(4), 243-280 (1983)

7. Looney, C.G., et al.: Cognitive situation and threat assessments of ground battlespaces. Information Fusion 4, 297-308 (2003)

8. Looney, C.G.: Exploring fusion architecture for a common operational picture. J. Information Fusion (article in press)

9. Schank, R.: Dynamic memory: a theory of reminding and learning in computers and people. Cambridge University Press, UK (1982)

10. Steinberg, A.N., et al.: Revisions to the JDL data fusion model. In: Proceedings of the SPIE. Sensor Fusion: Architectures, Algorithms and Applications, pp. 430-441 (1999) 